\title{
On the nullspace of arc-transitive graphs over finite fields
}

\author{
Primož Potočnik • Pablo Spiga • Gabriel Verret
}

Received: 12 February 2011 / Accepted: 5 December 2011 / Published online: 16 December 2011

(C) Springer Science+Business Media, LLC 2011

\begin{abstract}
Let $A$ be the adjacency matrix of a graph $\Gamma$. The nullity of $A$ (that is, the dimension of the nullspace of $A$ ), when viewed as a matrix over a field of prime characteristic $p$, is called the $p$-nullity of $\Gamma$. We present several families of arc-transitive graphs with arbitrarily large $p$-nullity. We also show that the $p$-nullity of a vertextransitive graph of order a power of $p$ is zero, provided that the valency of the graph is coprime to $p$.
\end{abstract}

Keywords Arc-transitive graphs · Graph-restrictive groups · Spectral graph theory · Finite fields

\section{Introduction}

Spectral graph theory is a well-developed area of research with fascinating applications in other areas of graph theory. In view of the vast amount of work done in this field, it is somewhat surprising that the spectrum of a graph is almost exclusively studied over a field of characteristic zero. There are at least two exceptions. Namely, in $[3,4]$ and [12], the rank of the adjacency matrix of a graph over the field of order 2 is used to bound its chromatic number. Furthermore, in the theory of association schemes and coherent configurations, the adjacency matrix of graphs and designs is

\footnotetext{
P. Potočnik

Faculty of Mathematics and Physics, University of Ljubljana, Ljubljana, Slovenia

P. Spiga (凶)

Dipartimento di Matematica ed Applicazioni, University of Milano-Bicocca, Via Cozzi 53, 20125

Milano, Italy

e-mail: pablo.spiga@unimib.it

P. Potočnik · G. Verret

Institute of Mathematics, Physics, and Mechanics, Jadranska 19, 1000 Ljubljana, Slovenia
} 
studied in detail over an arbitrary field to deduce some important facts about special families of graphs, for example strongly regular graphs. For this remarkable aspect of spectral graph theory, we refer the reader to $[2,6]$ and to the excellent recent survey article [5].

Our original motivation for this investigation was a seemingly unrelated question about the order of vertex-stabilisers in arc-transitive graphs [10]. The proof of a critical result required the existence of certain families of arc-transitive graphs with adjacency matrices having arbitrary large nullity over the field with $p$ elements. However, whilst searching for such graphs, the authors became fascinated by the topic and its plentiful connections with other areas of mathematics, ranging from projective geometry to number theory. The aim of this paper is thus two-fold; first, to convey some of our fascination and perhaps initiate further research in this area and, second, to construct a family of graphs solving our original problem. Before stating the main results, let us first introduce some notation which will be used throughout the paper.

Unless otherwise noted, graphs are finite and simple. Let $\Gamma$ be such a graph. An $s$-arc of $\Gamma$ is a sequence $\alpha=\left(v_{0}, \ldots, v_{s}\right)$ of $s+1$ vertices of $\Gamma$ such that each two consecutive vertices are adjacent and each three consecutive vertices in the sequence are pairwise distinct. A 1-arc is simply called an arc.

Let $G \leq \operatorname{Aut}(\Gamma)$ be a group of automorphisms of $\Gamma$. We say that $\Gamma$ is $G$-vertextransitive or $G$-arc-transitive if $G$ acts transitively on the vertices or the arcs of $\Gamma$, respectively. Similarly, we say that $\Gamma$ is $(G, s)$-arc-transitive if $G$ acts transitively on the set of $s$-arcs of $\Gamma$. We also say that $\Gamma$ is $(G, s)$-arc-regular if $G$ acts regularly on the set of $s-\operatorname{arcs}$ of $\Gamma$. When $G=\operatorname{Aut}(\Gamma)$, the prefix $G$ in the above definitions is usually omitted.

Let $p$ be a prime, let $\mathbb{F}$ be a field of characteristic $p$ and let $A$ be an integer-valued matrix. Taking $A$ over $\mathbb{F}$ simply means reducing the entries of $A$ modulo $p$. Note that this does not depend on the choice of $\mathbb{F}$. The rank of $A$ over $\mathbb{F}$ will be called the $p$-rank of $A$. Define the $p$-rank of a graph to be the $p$-rank of its adjacency matrix. Recall that the nullity of a matrix is the dimension of its nullspace. In an analogous manner as above, we define the p-nullity of an integer-valued matrix and of a graph.

Definition 1 Let $p$ be a prime and let $\mathcal{F}$ be a class of graphs. If, for every integer $M$, there exists a graph $\Gamma$ of $\mathcal{F}$ with $p$-nullity at least $M$, we say that $\mathcal{F}$ has unbounded p-nullity.

In this paper, we exhibit a few interesting such classes of graphs, which are summarised in the following theorem.

Theorem 2 Let $d \geq 3$ be an integer and let $p$ be a prime. The following families have unbounded p-nullity.

(1) connected 4-arc-transitive graphs,

(2) connected 4-arc-transitive 3-valent graphs, for $p=2$,

(3) connected arc-transitive graphs of valency $d$,

(4) the class of connected 3-valent graphs for which there exists a 2-arc-regular group of automorphisms containing a 1-arc-regular subgroup. 
Here are a few comments on the above theorem. First, note that part (3) improves part (1) for the class of arc-transitive graphs. Similarly, part (2) improves parts (1) and (3) but only for $p=2$ and $d=3$, respectively. Part (4) is the result needed to solve the problem which originally motivated our investigation. Let us briefly present this problem and its connection with the above theorem.

For a $G$-arc-transitive graph $\Gamma$ and a vertex $v \in V(\Gamma)$, let $G_{v}^{\Gamma(v)}$ be the permutation group induced by the action of the stabiliser $G_{v}$ on the neighbourhood $\Gamma(v)$ of the vertex $v$. A transitive permutation group $L$ is said to be graph-restrictive [11, Definition 2] provided that there exists a constant $c(L)$ such that, if $\Gamma$ is a connected $G$-arc-transitive graph with $G_{v}^{\Gamma(v)}$ permutation isomorphic to $L$ and $(u, v)$ is an arc of $\Gamma$, then $\left|G_{u v}\right| \leq c(L)$. By the classical result of Tutte on 3-valent arc-transitive graphs [15], transitive permutation groups of degree 3 are graph-restrictive (and the constant $c(L)$ can be chosen to be 16). Similarly, it is well known that, with the exception of the dihedral group $\mathrm{D}_{4}$ of degree 4 , all transitive permutation groups of degree 4 or 5 are graph-restrictive (see [16] for example). When trying to extend the classification of graph-restrictive groups to permutation groups of degree 6 , some key cases are: (i) the action of the alternating group $\mathrm{A}_{4}$ on the six unordered pairs of a 4-set, (ii) the action of the symmetric group $\mathrm{S}_{4}$ on the same six pairs and, (iii) the action of $\mathrm{S}_{4}$ on the cosets of a cyclic subgroup of order 4 . We are able to reduce the problem of proving that these permutation groups are not graph-restrictive to the problem of proving part (4) of Theorem 2 (see [10] for details).

Here is a brief summary of this paper. In Sects. 2 and 3 we give two simple constructions of 4-arc-transitive graphs proving parts (1) and (2) of Theorem 2. In Sect. 4, we set up some useful notation for graphs admitting a semiregular group of automorphisms. This is then used in Sects. 5 and 6 to prove, respectively, parts (3) and (4) of Theorem 2. Finally, in Sect. 7, we prove a remarkable result relating the $p$-nullity of a graph $\Gamma$ to the $p$-nullity of the quotient of $\Gamma$ by a semiregular group of automorphisms. As an application, we show that vertex-transitive graphs of valency $d$ and order a power of $p$ have trivial $p$-nullity if $\operatorname{gcd}(p, d)=1$.

\section{Incidence graphs of projective planes}

If there is no restriction on the valency, then it is not hard to exhibit examples of arc-transitive graphs with large $p$-nullity. Here is a well-studied example.

Proof of part (1) of Theorem 2 Let $a \in \mathbb{N}$, let $q=p^{a}$, and consider the incidence graph $\Gamma_{a}$ of the projective plane $\mathrm{PG}(2, q)$. The vertices of $\Gamma_{a}$ are the points and the lines of $\mathrm{PG}(2, q)$, with a point $P$ incident to a line $l$ in $\Gamma_{a}$ if and only if $P$ lies on $l$. It is known that $\Gamma_{a}$ is connected, is 4-arc-transitive (see [3, Sect. 5.3]) and its $p$-rank is $2\left(\left(\begin{array}{c}p+1 \\ 2\end{array}\right)^{a}+1\right)($ see $[13])$. Note that $\left(\begin{array}{c}p+1 \\ 2\end{array}\right)^{a}+1 \leq q^{2}$. Since $\Gamma_{a}$ has $2\left(q^{2}+q+1\right)$ vertices, it follows that $\Gamma_{a}$ has $p$-nullity at least $2(q+1)$. In particular, $\left\{\Gamma_{a} \mid a \in \mathbb{N}\right\}$ has unbounded $p$-nullity, proving part (1) of Theorem 2. 


\section{4-arc-transitive 3-valent graphs}

In this section, we study the 2-nullity of some arc-transitive 3-valent graphs. Our first observation is that this is always even. This follows from the fact that the number of vertices of a 3-valent graph is even and the 2-rank of a graph is even. Indeed, the adjacency matrix of a graph over a field of characteristic 2 can be viewed as the matrix of an alternating bilinear form, which is well known to have even rank (see [3, Theorem 8.10.1]).

We prove part (2) of Theorem 2, that is, that the class of connected 4-arc-transitive 3 -valent graphs has unbounded 2-nullity. We will require the following lemma, which is well known (see [12, Theorem 22]). We include a proof for completeness.

Lemma 3 Let $A$ be the adjacency matrix of a graph $\Gamma$ and let $\mu$ be the number of perfect matchings of $\Gamma$. Then $\operatorname{det} A$ has the same parity as $\mu$.

Proof Let $n$ be the number of vertices of $\Gamma$ and write $A=\left(a_{i j}\right)_{i, j}$. We have $\operatorname{det} A=$ $\sum_{\sigma} \operatorname{sgn}(\sigma) a_{11^{\sigma}} \cdots a_{n n^{\sigma}}$. Since we are only interested in the parity of $\operatorname{det} A$, we can omit $\operatorname{sgn}(\sigma)$. Moreover, $\sigma$ does not contribute to the sum unless $a_{11^{\sigma}} \cdots a_{n n^{\sigma}}=1$. Since $A$ is symmetric, if $a_{11^{\sigma}} \cdots a_{n n^{\sigma}}=1$, then $a_{11^{\sigma^{-1}}} \cdots a_{n n^{\sigma^{-1}}}=1$. In particular, if $\sigma \neq \sigma^{-1}$, then the contributions of $\sigma$ and $\sigma^{-1}$ to $\operatorname{det} A$ cancel each other. Therefore, we only need to consider the permutations $\sigma$ such that $\sigma^{2}=1$ and $a_{11^{\sigma}} \cdots a_{n n^{\sigma}}=1$. Clearly, each such permutation $\sigma$ gives rise to the perfect matching $\left\{\left\{i, i^{\sigma}\right\}\right\}_{i}$ of $\Gamma$. Conversely, each perfect matching of $\Gamma$ yields an involution $\sigma$ with $a_{11^{\sigma}} \cdots a_{n n^{\sigma}}=1$. This completes the proof of the lemma.

We also need the following simple result.

Lemma 4 Let $\Gamma$ be a connected 3-arc-transitive graph of valency $d \geq 2$ and let $\mu$ be the number of perfect matchings of $\Gamma$. Then $d(d-1)$ divides $\mu$. In particular, $\mu$ is even.

Proof Let $(u, v, w, x)$ be a 3 -arc of $\Gamma$. We first consider the degenerate case when $u=x$. By the 3-arc-transitivity of $\Gamma$, each 3-arc of $\Gamma$ is a 3-cycle and hence $\Gamma$ is a complete graph on 3 vertices, which admits no perfect matching. In particular, $\mu=0$ and the lemma follows. We may thus assume that $u \neq x$. Let $\mu_{1}$ be the number of perfect matchings containing $(u, v)$ and let $\mu_{2}$ be the number of perfect matchings containing both $(u, v)$ and $(w, x)$. In a perfect matching containing $(u, v)$, the vertex $w$ must be matched to any of its remaining $d-1$ neighbours. Since $\Gamma$ is 3 -arctransitive, each such choice leads to the same number of perfect matchings and hence $\mu_{1}=(d-1) \mu_{2}$. Similar arguments show that $\mu=d \mu_{1}$. Hence $\mu=d(d-1) \mu_{2}$ and $d(d-1)$ divides $\mu$.

Combining the above two lemmas, we get the following corollary.

Corollary 5 If $\Gamma$ is a connected 3-arc-transitive graph of valency $d \geq 2$, then $\Gamma$ has non-zero 2-nullity. 
Proof From Lemma 4, the number of perfect matchings of $\Gamma$ is even, and hence from Lemma 3, the determinant of the adjacency matrix of $\Gamma$ is even.

Proof of part (2) of Theorem 2 We construct an infinite family of connected 4-arctransitive 3-valent graphs. First, we point out that, by Dirichlet's theorem on primes in arithmetic progression, there exist infinitely many primes congruent to \pm 1 mod 16 . Let $r$ be such a prime and let $G_{r}=\operatorname{PSL}(2, r)$.

It is well known that $G_{r}$ contains a maximal subgroup $H$ isomorphic to $\mathrm{S}_{4}$ (see [14, Chap. 3, Sect. 6]). Moreover, in its action on the coset space $\Omega=G_{r} / H$, the group $G_{r}$ has a self-paired suborbit of size 3. In particular, $G_{r}$ is a group of automorphisms of an arc-transitive 3-valent graph $\Gamma_{r}$. Since $|H|=24$, the group $G_{r}$ acts 4-arc-transitively on $\Gamma_{r}$. As $G_{r}$ acts primitively on $\mathrm{V} \Gamma_{r}$, the graph $\Gamma_{r}$ is connected.

We claim that the 2-nullity of $\Gamma_{r}$ tends to infinity as $r$ tends to infinity. Let $V_{r}$ be the nullspace of the adjacency matrix $A_{r}$ of $\Gamma_{r}$ over $\mathbb{F}_{2}$. As $G_{r}$ acts as a group of automorphisms of $\Gamma_{r}$, we can view $V_{r}$ as a $G_{r}$-module. Since $G_{r}$ is a simple group, it either centralises or acts faithfully on $V_{r}$. If $G_{r}$ centralises $V_{r}$, then, since $G_{r}$ acts transitively on the vertices of $\Gamma_{r}$, the module $V_{r}$ must be a subspace of the one dimensional vector space spanned by the all-one vector $e$. Since $\Gamma_{r}$ is a 3-valent graph, we have $A_{r} e=3 e \neq 0$ and hence $e \notin V_{r}$. By Corollary 5, we know that $V_{r} \neq 0$ and hence $V_{r} \nsubseteq\langle e\rangle$. This shows that $G_{r}$ acts faithfully on $V_{r}$, that is, $G_{r}$ is isomorphic to a subgroup of $\operatorname{GL}\left(V_{r}\right)$. Since the order of $G_{r}$ tends to infinity with $r$, this implies that $\operatorname{dim} V_{r}$ also tends to infinity as $r$ tends to infinity, as claimed. In particular $\left\{\Gamma_{r} \mid\right.$ $r$ prime, $r \equiv \pm 1 \bmod 16\}$ is a family of connected 4-arc-transitive 3-valent graphs with unbounded 2-nullity.

\section{Graphs with semiregular groups of automorphisms}

In this section, we will consider the $p$-nullity of graphs which admit the action of a semiregular group of automorphisms. (A permutation group is said to be semiregular if the stabiliser of each point is trivial.) Note that the much studied family of Cayley graphs is a special case of this situation. The existence of a semiregular group of automorphisms $H$ of a graph $\Gamma$ allows a more compact representation of the adjacency matrix $A$ of $\Gamma$. Rather than considering $A$ as a matrix of dimension $|\mathrm{V}(\Gamma)|$ with coefficients in a field $\mathbb{F}$, we can consider it as a matrix of dimension $|\mathrm{V}(\Gamma)| /|H|$ with coefficients in the group algebra $\mathbb{F}[H]$. (See [7] for a good reference about this approach.)

As an application of this approach, we will prove three results, the first two dealing with the $p$-nullity of certain Cayley graphs (see Sect. 5 and Sect. 6) and the third dealing with the $p$-nullity of vertex-transitive graphs with a power of $p$ number of vertices (see Sect. 7). In the last of the three applications, it will prove useful to consider the $p$-nullity in the setting of multigraphs rather than graphs.

By a multigraph, we mean an ordered pair $\Gamma=(V, \mu)$, where $V$ is the set of vertices and $\mu: V \times V \rightarrow \mathbb{N}$ satisfies $\mu(u, v)=\mu(v, u)$ and is called the edgemultiplicity function. The valency of a vertex $v \in V$ is defined by $\sum_{w \in \mathrm{V}} \mu(\{w, v\})$. Note that every graph can be considered as a multigraph by setting $\mu(u, v)=1$ 
if $\{u, v\}$ is an edge and $\mu(u, v)=0$ otherwise. The adjacency matrix of $\Gamma$ is the $(|V| \times|V|)$-matrix whose rows and columns are indexed by elements of $V$ in which the $(u, v)$-entry equals $\mu(\{u, v\})$. An automorphism of the multigraph $\Gamma=(V, \mu)$ is a permutation of $V$ which preserves $\mu$.

If $\mathbb{F}[V]$ is the free $\mathbb{F}$-module over $V$ (that is, the vector space of all formal linear combinations of elements in $V$ with coefficients in the field $\mathbb{F}$ ), then the adjacency matrix $A$ may be viewed as the endomorphism of $\mathbb{F}[V]$ mapping a basis element $v \in V$ to the sum $\sum_{u \in V} \mu(\{v, u\}) u$. As in the case of graphs, a permutation $g$ of $V$ is an automorphism of $\Gamma$ if and only if the induced permutation representation of $g$ on $\mathbb{F}[V]$ commutes with $A$.

Suppose now that $\Gamma$ admits a group of automorphisms $H$ acting semiregularly on $V$. Let $P_{1}, \ldots, P_{k}$ denote the orbits of $H$ and choose a reference vertex $v_{i} \in P_{i}$, for each $i$. The semiregularity of $H$ allows us to identify each $P_{i}$ with a copy of $H$ (where $v_{i}$ gets identified with $1 \in H$ ), in such a way that the regular action of $H$ on $P_{i}$ is permutation isomorphic to the action of $H$ on itself by right multiplication. This identification, extended by linearity to $\mathbb{F}[V]$, defines an isomorphism $\iota$ of the space $\mathbb{F}[V]$ with

$$
\mathbb{F}[H]^{k}=\mathbb{F}[H] \oplus \cdots \oplus \mathbb{F}[H],
$$

the direct sum of $k=|V| /|H|$ copies of the group algebra $\mathbb{F}[H]$. The semiregular action of $h \in H$ on $\mathbb{F}[V]$ corresponds to the componentwise multiplication by the scalar $h \in \mathbb{F}[H]$ in $\mathbb{F}[H]^{k}$. In particular, $\mathbb{F}[V]$ is a free $\mathbb{F}[H]$-module. Also, the isomorphism $\iota$ identifies $\mathbf{M}_{k}(\mathbb{F}[H])$ with a subalgebra of $\mathbf{M}_{|V|}(\mathbb{F})$.

Since the action of the adjacency matrix $A$ on $\mathbb{F}[V]$ commutes with each $h \in H$, the $\mathbb{F}$-endomorphism $A$ of $\mathbb{F}[V]$ is also a $\mathbb{F}[H]$-endomorphism of the $\mathbb{F}[H]$-module $\mathbb{F}[V]$. Thus we can represent $A$ as a $(k \times k)$-matrix over $\mathbb{F}[H]$.

Observe that the $A$-image of the $i$ th standard basis vector $e_{i}$ of $\mathbb{F}[H]^{k}=\mathbb{F}[V]$ is precisely the row of $A$ indexed by the reference vertex $v_{i} \in P_{i}$. With respect to the standard basis $\left(e_{i}\right)_{i=1}^{k}$, the $j$ th component of the $i$ th row of $A$ (as an element of $\left.\mathbf{M}_{k}(\mathbb{F}[H])\right)$ equals

$$
\sum_{h \in H} \mu\left(\left\{v_{i}, v_{j}^{h}\right\}\right) h
$$

More precisely, we have shown the following.

Proposition 6 Let $\Gamma=(V, \mu)$ be a multigraph admitting a semiregular group of automorphisms $H$ having $k$ orbits on $V$. For each orbit $P_{i}$ of $H$ choose a vertex $v_{i} \in$ $P_{i}$, and consider the matrix $A \in \mathrm{M}_{k}(\mathbb{F}[H])$ with the $(i, j)$-entry being the sum (*) above. Then $A$ is the adjacency matrix of $\Gamma$.

This has the following straightforward consequence for Cayley graphs.

Corollary 7 Let $\Gamma=\operatorname{Cay}(H, S)$ be a Cayley graph. The adjacency matrix of $\Gamma$ is $\sum_{s \in S} s \in \mathbb{F}[H]$. Also, the nullity of $\Gamma$ over $\mathbb{F}$ is the dimension over $\mathbb{F}$ of the right annihilator of the element $\sum_{s \in S} s$ in the group algebra $\mathbb{F}[H]$. 
Proof We use Proposition 6. Since $H$ acts regularly on $\mathrm{V} \Gamma$, the group $H$ has only one orbit on $\mathrm{V} \Gamma$, with reference point 1 say. Since $S$ is the neighbourhood of 1 in $\Gamma$, we see that $\sum_{s \in S} \mu(\{1, s\}) s=\sum_{s \in S} s$ is the adjacency matrix of $\Gamma$. The rest of the corollary follows.

\section{Arc-transitive dihedrants}

In this section, we prove part (3) of Theorem 2. Recall that $d \geq 3$ and $p$ is a prime. Let $a=p^{k}$ for some $k \geq 1$ and let $n=1+a+a^{2}+\cdots+a^{d-1}$. Denote by $\mathrm{D}_{n}$ the dihedral group of order $2 n$ generated by $\{r, t\}$, where $r$ has order $n$ and $t$ has order 2. Let $S=\left\{r t, r^{a} t, r^{a^{2}} t, \ldots, r^{a^{d-1}} t\right\}$ and consider the dihedrant $\Gamma_{k}=\operatorname{Cay}\left(\mathrm{D}_{n}, S\right)$. Clearly, $\Gamma_{k}$ is a vertex-transitive graph with $2 n$ vertices and valency $d$.

We claim that $\Gamma_{k}$ is arc-transitive. Note that, since $\operatorname{gcd}(n, a)=1$, the function

$$
\varphi:\left\{\begin{array}{l}
r \mapsto r^{a}, \\
t \mapsto t
\end{array}\right.
$$

extends to an automorphism of $\mathrm{D}_{n}$ (which we still denote by $\varphi$ ) with $\langle\varphi\rangle$ acting transitively on the neighbours of 1 in $\Gamma_{k}$. Therefore $G=\mathrm{D}_{n} \rtimes\langle\varphi\rangle$ is an arc-transitive group of automorphisms of $\Gamma_{k}$ with $G_{1}=\langle\varphi\rangle \cong \mathbb{Z}_{d}$ and $\Gamma_{k}$ is an arc-transitive dihedrant.

Note that $\langle S\rangle=\left\langle r t, r^{a-1}\right\rangle=\left\langle r t, r^{\operatorname{gcd}(a-1, n)}\right\rangle$, which has index $\operatorname{gcd}(a-1, n)$ in $\mathrm{D}_{n}$. This shows that $\Gamma_{k}$ has $\operatorname{gcd}(a-1, n)=\operatorname{gcd}(a-1, d)$ connected components. In particular, the number of connected components of $\Gamma_{k}$ is at most $d$. In the rest of the proof, we study the $p$-nullity of $\Gamma_{k}$.

Proof of part (3) of Theorem 2 We use the dihedrants $\Gamma_{k}$ introduced above. Let $\bar{S}=\sum_{s \in S} s \in \mathbb{F}_{p}\left[\mathrm{D}_{n}\right]$. By Corollary 7 , the $p$-nullity of $\Gamma_{k}$ equals the dimension $\operatorname{dim}_{\mathbb{F}_{p}}(\operatorname{ann}(\bar{S}))$ over $\mathbb{F}_{p}$ of the right annihilator ann $(\bar{S})$ of $\bar{S}$ in the group algebra $\mathbb{F}_{p}\left[\mathrm{D}_{n}\right]$. As $t$ is a unit in the ring $\mathbb{F}_{n}\left[\mathrm{D}_{n}\right]$, we see that $\operatorname{ann}(\bar{S})=\operatorname{ann}(N)$, where $N=\sum_{l=0}^{d-1} r^{a^{l}}$.

Since the group algebra $\mathbb{F}_{p}\left[\mathrm{D}_{n}\right]$ splits into the direct sum $\mathbb{F}_{p}[\langle r\rangle] \oplus \mathbb{F}_{p}[\langle r\rangle] t$, if we let $\operatorname{ann}_{\langle r\rangle}(N)=\operatorname{ann}(N) \cap \mathbb{F}_{p}[\langle r\rangle]$, then $\operatorname{ann}(N)=\operatorname{ann}_{\langle r\rangle}(N) \oplus \operatorname{ann}_{\langle r\rangle}(N) t$. Hence $\operatorname{dim}_{\mathbb{F}_{p}}(\operatorname{ann}(N))$ equals twice the dimension of the right annihilator of $N$ in $\mathbb{F}_{p}[\langle r\rangle]$, which we will now compute.

Identifying the elements of $\langle r\rangle$ with the corresponding matrices in the right regular permutation representation $\langle r\rangle \rightarrow \operatorname{GL}\left(n, \mathbb{F}_{p}\right)$, we have to compute the dimension of the kernel of the $(n \times n)$-matrix $N=r+r^{a}+\cdots+r^{a^{d-1}}$.

Let $p_{r}(T)$ be the characteristic polynomial of $r \in \mathrm{GL}\left(n, \mathbb{F}_{p}\right)$ over $\mathbb{F}_{p}$. Clearly, as $r$ has order $n$, we have $p_{r}(T)=T^{n}-1$. Now, since $p_{r}(T)$ and $p_{r}^{\prime}(T)=n T^{n-1}=T^{n-1}$ are coprime, it follows that $p_{r}(T)$ has $n$ distinct roots in a suitable extension of $\mathbb{F}_{p}$, that is, $r$ has $n$ distinct eigenvalues in the algebraic closure of $\mathbb{F}_{p}$.

Note that the set of eigenvalues of $N=\sum_{l=0}^{d-1} r^{a^{l}}$ is $\left\{\sum_{l=0}^{d-1} \lambda^{a^{l}} \mid \lambda\right.$ eigenvalue of $\left.r\right\}$. Namely, if $\lambda$ is an eigenvalue of $r$ for the eigenvector $v$, then $\sum_{l=0}^{d-1} \lambda^{a^{l}}$ is an eigenvalue of $N$ for the eigenvector $v$. In particular, the $p$-nullity of $N$ is the number of 
common roots of $p_{r}(T)$ and $\sum_{l=0}^{d-1} T^{a^{l}}$. Write $g(T)=\sum_{l=0}^{d-1} T^{a^{l}}$ and null $(N)$ for the $p$-nullity of $N$.

Let $f(T)=T^{a^{d}}-T$. Consider $E=\mathbb{F}_{a^{d}}$ and $F=\mathbb{F}_{a}$ the fields with $a^{d}$ and $a$ elements, respectively. As $|E|=a^{d}$, the elements of $E$ are exactly the roots of the polynomial $f(T)$. Note that $g(T)^{a}=T^{a}+T^{a^{2}}+\cdots+T^{a^{d}}$ and hence $g(T)\left(g(T)^{a-1}-1\right)=g(T)^{a}-g(T)=T^{a^{d}}-T=f(T)$. In particular, the polynomial $g(T)$ divides $f(T)$ and hence the roots of $g(T)$ are elements of $E$. Moreover, since $n$ divides $a^{d}-1$, we find that $p_{r}(T)$ divides $f(T)$ and hence the roots of $p_{r}(T)$ lie in $E$. This shows that the roots of $p_{r}(T)$ and $g(T)$ are elements of $E$.

We claim that the common roots of $p_{r}(T)$ and $g(T)$ are the elements of $E$ of norm 1 and trace 0 in the Galois extension $E / F$. Indeed, if $x \in E$, then the norm of $x$ in $E / F$ is

$$
\mathrm{N}_{E / F}(x)=\prod_{l=0}^{d-1} x^{a^{l}}=x^{\sum_{l=0}^{d-1} a^{l}}=x^{n}
$$

and $\mathrm{N}_{E / F}(x)=1$ if and only if $p_{r}(x)=0$. Similarly, the trace of $x$ in $E / F$ is

$$
\operatorname{Tr}_{E / F}(x)=\sum_{l=0}^{d-1} x^{a^{l}}=g(x)
$$

and $\operatorname{Tr}_{E / F}(x)=0$ if and only if $g(x)=0$. Now, Moisio in [9, Sect. 3] obtains tight upper and lower bounds on the number of field elements in the finite extension $E / F$ of norm 1 and trace 0 , in particular from [9, Corollary 3.3] we obtain

$$
(\dagger) \quad \operatorname{null}(N) \geq \frac{a^{d-1}-1}{a-1}-\operatorname{gcd}(a-1, d) a^{(d-2) / 2} .
$$

Denote by $\Gamma_{k}^{1}$ the connected component of $\Gamma_{k}$ containing 1 and consider the family $\left\{\Gamma_{k}^{1} \mid k \in \mathbb{N}\right\}$. By construction $\Gamma_{k}^{1}$ is a connected arc-transitive graph of valency $d$. As $\Gamma_{k}$ has $\operatorname{gcd}(a-1, d)$ connected components, from $(\dagger)$ we see that $\Gamma_{k}^{1}$ has $p$ nullity at least $\frac{a^{d-1}-1}{\operatorname{gcd}(a-1, d)(a-1)}-a^{(d-2) / 2}$. In particular, the $p$-nullity of $\Gamma_{k}^{1}$ tends to infinity as $k$ tends to infinity and the proof is complete.

Remark This proof of part (3) of Theorem 2 depends on deep number theoretic results about Kloosterman sums from [9, Sect. 3]. For $d>3$, it is possible to deduce that the number of common roots of $p_{r}(T)$ and $g(T)$ tends to infinity as $a$ tends to infinity by using the Stepanov-Schmidt method and a theorem of A. Weil, (see [8] for a general account of the Stepanov-Schmidt method and [8, Theorem 6.61] for our particular application). This yields another proof of part (3) of Theorem 2 (for $d>3)$.

\section{2-arc-regular 3-valent generalised dihedrants}

This section is devoted to the proof of part (4) of Theorem 2. In particular, we will construct an infinite family of connected 3-valent Cayley graphs $\Gamma$, admitting a 2- 
arc-regular group of automorphisms $A$ which contains a 1-arc-regular subgroup $\bar{A}$. We will then show that this family has an unbounded $p$-nullity for each prime $p$. We will make use of the theory developed in Sect. 4.

Construction 8 Let $p$ be a prime and let $n$ be a natural number. Let $G_{n}$ be the group $(\langle i\rangle \times\langle j\rangle \times\langle g\rangle) \rtimes\langle h\rangle$, where $|i|=|j|=p^{n},|g|=3,|h|=2, i^{h}=i^{-1}, j^{h}=j^{-1}$ and $g^{h}=g^{-1}$. (Such a group is sometimes called a generalised dihedral group over the abelian group $\mathbb{Z}_{p^{n}} \times \mathbb{Z}_{p^{n}} \times \mathbb{Z}_{3}$.) Let $S_{n}=\left\{h, i g h, j g^{2} h\right\}$ and let $\Gamma_{n}=\operatorname{Cay}\left(G_{n}, S_{n}\right)$.

Note that $S_{n}$ consists of three involutions and hence $\Gamma_{n}$ is a vertex-transitive 3valent graph with $6 p^{2 n}$ vertices. It is not hard to see that, if $p \neq 3$, then $G_{n}=$ $\left\langle h, i g h, j g^{2} h\right\rangle$ and $\Gamma_{n}$ is connected, whilst if $p=3$, then $\Gamma_{n}$ has 3 connected components.

We claim that $\Gamma_{n}$ admits a group of automorphisms $A_{n}$ acting 2-arc-regularly, and containing a subgroup $\overline{A_{n}}$ acting arc-regularly.

We leave to the reader to check that the map

$$
\alpha:\left\{\begin{array}{l}
i \mapsto j, \\
j \mapsto i, \\
g \mapsto g^{2}, \\
h \mapsto h
\end{array}\right.
$$

determines an automorphism of $G_{n}$ fixing $h$ and swapping $i g h$ and $j g^{2} h$. Similarly, the map

$$
\beta:\left\{\begin{array}{l}
i \mapsto i^{-1} j, \\
j \mapsto i^{-1}, \\
g \mapsto g, \\
h \mapsto i g h
\end{array}\right.
$$

determines an automorphism of $G_{n}$ acting as a 3-cycle on $S_{n}$. Since the automorphisms $\alpha$ and $\beta$ of $G_{n}$ fix $S_{n}$ setwise, the groups $\overline{A_{n}}=G_{n} \rtimes\langle\beta\rangle$ and $A_{n}=$ $G_{n} \rtimes\langle\alpha, \beta\rangle$ act arc-regularly and 2-arc-regularly on $\Gamma_{n}$, respectively, with $\overline{A_{n}} \subseteq A_{n}$, as required.

Proposition 9 Let $p$ be a prime and let $n$ be a natural number. The p-nullity of the graph $\Gamma_{n}$ from Construction 8 is at least $4 p^{n}$.

Proof Throughout the proof, we use the notation of Construction 8 but we drop the subscript $n$, writing $G$ for $G_{n}, S$ for $S_{n}$ and $\Gamma$ for $\Gamma_{n}$.

Let $\mathbb{F}=\mathbb{F}_{p}$ be the field of cardinality $p$. By a slight abuse of notation, we interpret $S$ as the element $h+i g h+j g^{2} h$ of the group algebra $\mathbb{F}[G]$. In view of Corollary 7 , the $p$-nullity of $\Gamma$ equals the dimension $\operatorname{dim}_{\mathbb{F}}\left(\operatorname{ann}_{\mathbb{F}[G]}(S)\right)$ over $\mathbb{F}$ of the right annihilator of $S$ in the ring $\mathbb{F}[G]$. As $h$ is a unit of $\mathbb{F}[G]$, we see that $\operatorname{ann}_{\mathbb{F}[G]}(S)=\operatorname{ann}_{\mathbb{F}[G]}(N)$, where $N=1+i g+j g^{2} \in \mathbb{F}[G]$. 
Let $H=\langle i, j, g\rangle$ and observe that the group algebra $\mathbb{F}[G]$ splits (as a left $\mathbb{F}[H]$ module) into the direct sum $\mathbb{F}[H] \oplus \mathbb{F}[H] h$. Moreover, since $N \in \mathbb{F}[H]$, it follows that $\operatorname{ann}_{\mathbb{F}[G]}(N)=\operatorname{ann}_{\mathbb{F}[H]}(N) \oplus \operatorname{ann}_{\mathbb{F}[H]}(N) h$, and hence the $\mathbb{F}$-dimension of $\operatorname{ann}_{\mathbb{F}[G]}(N)$ equals twice the $\mathbb{F}$-dimension of the right annihilator $\operatorname{ann}_{\mathbb{F}[H]}(N)$ of $N$ in $\mathbb{F}[H]$. To prove the proposition it therefore suffices to show that $\operatorname{dim}_{\mathbb{F}}\left(\operatorname{ann}_{\mathbb{F}[H]}(N)\right) \geq$ $2 p^{n}$.

Write $e=1+g+g^{2}$ if $p \neq 3$ and $e=0$ if $p=3$. Since $\mathbb{F}$ has characteristic $p$ and $H$ is abelian, we have

$$
N^{p^{n}}=1^{p^{n}}+(i g)^{p^{n}}+\left(j g^{2}\right)^{p^{n}}=1+g^{p^{n}}+g^{2 p^{n}}=e .
$$

Hence $\operatorname{ann}_{\mathbb{F}[H]}(N) \subseteq \operatorname{ann}_{\mathbb{F}[H]}\left(N^{p^{n}}\right)=\operatorname{ann}_{\mathbb{F}[H]}(e)$. Thus $\operatorname{ann}_{\mathbb{F}[H]}(N)$ is equal to the kernel of the linear map

$$
\tilde{N}: \operatorname{ann}_{\mathbb{F}[H]}(e) \rightarrow \operatorname{ann}_{\mathbb{F}[H]}(e), \quad \tilde{N}: x \mapsto N x .
$$

Now we compute the $\mathbb{F}$-dimension of $\operatorname{ann}_{\mathbb{F}[H]}(e)$. If $p=3$, then $e=0$ and hence $\operatorname{dim}_{\mathbb{F}}\left(\operatorname{ann}_{\mathbb{F}[H]}(e)\right)=3 p^{2 n}$. If $p \neq 3$, then we show that $\operatorname{dim}_{\mathbb{F}}\left(\operatorname{ann}_{\mathbb{F}[H]}(e)\right)=2 p^{2 n}$. Let $L=\langle i, j\rangle$ and let $w$ be an arbitrary element of $\mathbb{F}[H]$. Then $w$ can be written uniquely as $w=x+y g+z g^{2}$ for some $x, y, z \in \mathbb{F}[L]$. We have $e w=(1+g+$ $\left.g^{2}\right)\left(x+y g+z g^{2}\right)=(x+y+z)+(x+y+z) g+(x+y+z) g^{2}$. Since $\mathbb{F}[H]=$ $\mathbb{F}[L] \oplus \mathbb{F}[L] g \oplus \mathbb{F}[L] g^{2}$, it follows that $w \in \operatorname{ann}_{\mathbb{F}[H]}(e)$ if and only if $x+y+z=0$. We conclude that $\operatorname{ann}_{\mathbb{F}[H]}(e)$ has $\mathbb{F}$-dimension $2 p^{2 n}$. Note that in both cases $(p=3$ and $p \neq 3$ ), it follows that $\operatorname{dim}_{\mathbb{F}}\left(\operatorname{ann}_{\mathbb{F}[H]}(e)\right) \geq 2 p^{2 n}$.

Notice that $N^{p^{n}}=e$ implies that $\tilde{N}^{p^{n}}=0$ and hence each Jordan block of $N$ has size at most $p^{n}$. It follows that the kernel of $\tilde{N}$ has dimension at least $2 p^{2 n} / p^{n}=2 p^{n}$. To conclude the proof, recall that the $p$-nullity of $\Gamma$ is twice the $\mathbb{F}$-dimension of $\operatorname{ann}_{\mathbb{F}[H]}(N)$.

Proof of part (4) of Theorem 2 Using the notation from Construction 8, we already remarked that $\Gamma_{n}$ is a 3-valent graph admitting a 2-arc-regular group of automorphisms with a 1-arc-regular subgroup. Moreover, if $p \neq 3$, then $\Gamma_{n}$ is connected and hence, from Proposition 9, we see that $\left\{\Gamma_{n} \mid n \in \mathbb{N}\right\}$ is a family of graphs satisfying the hypothesis of part (4) of Theorem 2 and with unbounded $p$-nullity.

If $p=3$, then $\Gamma_{n}$ has 3 connected components. Denote one of these connected components by $\Gamma_{n}^{\prime}$. Clearly, $\Gamma_{n}^{\prime}$ also admits a 2-arc-regular group of automorphisms with a 1 -arc-regular subgroup. From Proposition 9 , we find that $\Gamma_{n}^{\prime}$ has $p$-nullity at least $\left(4 \cdot 3^{n}\right) / 3=4 \cdot 3^{n-1}$ and hence $\left\{\Gamma_{n}^{\prime} \mid n \in \mathbb{N}\right\}$ is a family of graphs satisfying the hypothesis of part (4) of Theorem 2 and with unbounded $p$-nullity.

\section{Vertex-transitive graphs of prime power order}

In contrast to the previous sections, where we were considering families of graphs with large $p$-nullity, this section is devoted to vertex-transitive graphs with trivial $p$ nullity. In particular, we will show that the $p$-nullity of a vertex-transitive graph on a power of $p$ of vertices is zero provided that the valency of $\Gamma$ is not divisible by $p$. 
The main idea of the proof is based on the fact that such graphs admit a semiregular group of automorphisms of order $p$. We will use this group to reduce the problem to a smaller (multi)graph with the same properties and then proceed by induction. Besides the theory developed in Sect. 4 we will also need a result concerning quotient multigraphs, which we now briefly describe.

Let $H$ be a group of automorphisms of a multigraph $\Gamma=(V, \mu)$ and let $\mathcal{P}=$ $\left\{P_{1}, \ldots, P_{k}\right\}$ be the partition of $V$ into orbits of $H$. For each $i$, choose a reference vertex $v_{i} \in P_{i}$. We define the quotient multigraph $\Gamma / H$ as the multigraph with vertex-set $\mathcal{P}$ and the edge-multiplicity of $\{P, Q\}$ in $\Gamma / H$ is defined as the sum of the multiplicities $\mu(\{u, v\})$, where $u$ is a fixed vertex of $P$ and $v$ runs through the neighbours of $u$ in $Q$. Note that this sum is independent of the choice of the vertex $u$ in $P$, and that we get the same value for the edge-multiplicity if we swap the roles of $P$ and $Q$.

By the definition of the quotient multigraph $\Gamma / H$, the adjacency matrix $A^{\prime}$ of $\Gamma / H$ is a $(k \times k)$-matrix with rows and columns indexed by the orbits $P_{1}, \ldots, P_{k}$ of $H$, where the $\left(P_{i}, P_{j}\right)$-entry equals the sum

$$
\sum_{u \in P_{j}} \mu\left(\left\{v_{i}, u\right\}\right)=\sum_{h \in H} \mu\left(\left\{v_{i}, v_{j}^{h}\right\}\right) .
$$

Note that the latter is precisely the value obtained from the $(i, j)$-entry of $A$ (viewed as an element of $\mathbf{M}_{k}(\mathbb{F}[H])$ ) by applying the augmentation homomorphism $\varphi$ : $\mathbb{F}[H] \rightarrow \mathbb{F}$, mapping each $h \in H$ to 1 . We have thus proved the following interesting fact.

Proposition 10 Let $H$ be a semiregular group of automorphisms of a multigraph $\Gamma$ and let $A$ be the adjacency matrix of $\Gamma$, viewed as a $(k \times k)$-matrix over $\mathbb{F}[H]$. Then the adjacency matrix of the quotient multigraph $\Gamma / H$ is the matrix obtained from $A$ by applying the ring homomorphism $\hat{\varphi}: \mathrm{M}_{k}(\mathbb{F}[H]) \rightarrow \mathrm{M}_{k}(\mathbb{F})$ induced entry-wise by the augmentation homomorphism $\varphi: \mathbb{F}[H] \rightarrow \mathbb{F}$.

Let us now prove the following simple lemma concerning local rings (i.e. rings with a unique maximal ideal).

Lemma 11 Let $R$ and $S$ be commutative local rings and let $\varphi: R \rightarrow S$ be a surjective ring homomorphism. Let $\hat{\varphi}$ be the homomorphism from $\mathrm{M}_{n}(R)$ to $\mathrm{M}_{n}(S)$ induced by $\varphi$ and let $A \in \mathrm{M}_{n}(R)$. Then, $A$ is invertible in $\mathrm{M}_{n}(R)$ if and only if $A^{\hat{\varphi}}$ is invertible in $\mathrm{M}_{n}(S)$.

Proof Recall that the set of invertible elements in a local ring is precisely the complement of the maximal ideal. Since a surjective ring homomorphism maps maximal ideals to maximal ideals, this shows that an element $r \in R$ is invertible in $R$ if and only if $r^{\varphi}$ is invertible in $S$. To conclude the proof, note that $\operatorname{det}\left(A^{\hat{\varphi}}\right)=(\operatorname{det} A)^{\varphi}$ and that a matrix is invertible if and only if its determinant is invertible.

Finally, we prove the following key result relating the nullity of a multigraph with that of its quotient under an abelian $p$-group. 
Proposition 12 Let $\Gamma$ be a vertex-transitive multigraph, let $\mathbb{F}$ be a field of characteristic $p$ and let $C$ be an abelian p-group of automorphisms of $\Gamma$, acting semiregularly on the vertices. Then, the adjacency matrix of $\Gamma$ is invertible over $\mathbb{F}$ if and only if the adjacency matrix of $\Gamma / C$ is invertible over $\mathbb{F}$.

Proof Let $k$ be the number of orbits of $C$ on the vertices of $\Gamma$, let $A$ be the adjacency matrix of $\Gamma$, viewed as a $(k \times k)$-matrix over $\mathbb{F}[C]$, and let $A_{C}$ be the adjacency matrix of $\Gamma / C$. In view of Proposition 10 , we have $A_{C}=A^{\hat{\varphi}}$, where $\hat{\varphi}$ denotes the mapping induced by the augmentation ring homomorphism $\varphi: \mathbb{F}[C] \rightarrow \mathbb{F}$.

Since $C$ is a $p$-group and $\mathbb{F}$ has characteristic $p, \mathbb{F}[C]$ is a local ring (see $[1$, Corollary 3, Chap. I]). Hence, by Lemma $11, A_{C}=A^{\hat{\varphi}}$ is invertible if and only if $A$ is invertible.

We conclude the paper with a nice application of Proposition 12.

Theorem 13 Let $p$ be a prime and let $\Gamma$ be a vertex-transitive multigraph of valency $d$ on $n$ vertices. Let $\mathbb{F}$ be a field of characteristic $p$. If $\operatorname{gcd}(p, d)=1$ and $n$ is a power of $p$, then the adjacency matrix of $\Gamma$ is invertible over $\mathbb{F}$.

Proof The proof goes by induction on $n$. If $n=1$, then $\Gamma$ consists of a single vertex with $d$ loops and its adjacency matrix is the $(1 \times 1)$-matrix $[d]$. This matrix is invertible over $\mathbb{F}$ since $\operatorname{gcd}(p, d)=1$.

We now assume that $n>1$. Let $G=\operatorname{Aut}(\Gamma)$ and let $P$ be a Sylow $p$-subgroup of $G$. Since $n$ is a power of $p$, the group $P$ acts transitively on the vertices of $\Gamma$. Let $C$ be a central subgroup of $P$ with $|C|=p$. Since $C$ is central in $P$, it must act semiregularly on the vertices of $\Gamma$. Consider $\Gamma / C$. This is a vertex-transitive multigraph of valency $d$ and $|\mathrm{V}(\Gamma / C)|$ is a strict divisor of $n$. By the induction hypothesis, the adjacency matrix of $\Gamma / C$ is invertible over $\mathbb{F}$. Proposition 12 then completes the induction step and the proof.

Acknowledgements We wish to thank Giuseppe Marino for pointing out and discussing the results in [9]. The second author is supported by UWA as part of the Australian Council Federation Fellowship Project FF0776186.

\section{References}

1. Alperin, J.L.: Local Representation Theory. Cambridge Studies in Advanced Mathematics, vol. 11. Cambridge University Press, Cambridge (1986)

2. Brouwer, A.E., van Eijl, C.A.: On the $p$-rank of the adjacency matrices of strongly regular graphs. J. Algebr. Comb. 1, 329-346 (1992)

3. Godsil, C., Royle, G.: Algebraic Graph Theory. Graduate Texts in Mathematics, vol. 207. Springer, New York (2001)

4. Godsil, C., Royle, G.: Chromatic number and the 2-rank of a graph. J. Comb. Theory, Ser. B 81, 142-149 (2001)

5. Haemers, W.H.: Matrices for graphs, designs and codes. In: Crnković, D., Tonchev, V. (eds.) Information Security, Coding Theory and Related Combinatorics, pp. 253-277. IOS Press, Amsterdam (2011)

6. Haemers, W.H., Peeters, R., van Rijckevorsel, J.M.: Binary codes of strongly regular graphs. Des. Codes Cryptogr. 17, 187-209 (1999) 
7. Kovács, I., Malnič, A., Marusič, D., Miklavič, S., Transitive group actions: (im)primitivity and semiregular subgroups. arXiv:math/0701686v1 [math.GR]

8. Lidl, R., Niederreiter, H.: Finite Fields. Encyclopedia of Mathematics and Its Applications. Cambridge University Press, Cambridge (1984)

9. Moisio, M.M.: Kloosterman sums, elliptic curves, and irreducible polynomials with prescribed trace and norm. Acta Arith. 134, 329-349 (2008)

10. Potočnik, P., Spiga, P., Verret, G.: An explicit method for constructing arc-transitive graphs with unbounded vertex stabilisers (in preparation)

11. Potočnik, P., Spiga, P., Verret, G.: On graph-restrictive permutation groups. arXiv:1101.5186v2 [math.CO]

12. Rotman, J.J.: Projective planes, graphs, and simple algebras. J. Algebra 155, 267-289 (1993)

13. Smith, K.J.C.: On the $p$-rank of the incidence matrix of points and hyperplanes in a finite projective geometry. J. Comb. Theory 7, 122-129 (1969)

14. Suzuki, M.: Group Theory I. Springer, New York (1982)

15. Tutte, W.T.: A family of cubical graphs. Proc. Camb. Philos. Soc. 43, 459-474 (1947)

16. Weiss, R.: Presentation for $(G, s)$-transitive graphs of small valency. Math. Proc. Philos. Soc. 101, 7-20 (1987) 